\title{
APEX1 Polymorphisms and Neuroblastoma Risk in Chinese Children: A Three-Center Case-Control Study
}

\author{
Jiabin Liu $\mathbb{D},{ }^{1}$ Wei Jia $\mathbb{D}^{1},{ }^{1}$ Rui-Xi Hua $\mathbb{D}^{2},{ }^{2}$ Jinhong Zhu $\mathbb{D},{ }^{3}$ Jiao Zhang, ${ }^{4}$ Tianyou Yang $\mathbb{D},{ }^{1}$ \\ Peng Li, ${ }^{5}$ Huimin Xia $\mathbb{1},{ }^{1}$ Jing $\mathrm{He}\left(\mathbb{B},{ }^{1}\right.$ and Jiwen Cheng ${ }^{5}{ }^{5}$ \\ ${ }^{1}$ Department of Pediatric Surgery, Guangzhou Institute of Pediatrics, Guangzhou Women and Children's Medical Center, \\ Guangzhou Medical University, Guangzhou, 510623 Guangdong, China \\ ${ }^{2}$ Department of Oncology, The First Affiliated Hospital of Sun Yat-sen University, Guangzhou, 510080 Guangdong, China \\ ${ }^{3}$ Department of Clinical Laboratory, Molecular Epidemiology Laboratory, Harbin Medical University Cancer Hospital, Harbin, \\ 150040 Heilongjiang, China \\ ${ }^{4}$ Department of Pediatric Surgery, The First Affiliated Hospital of Zhengzhou University, Zhengzhou, 450052 Henan, China \\ ${ }^{5}$ Department of Pediatric Surgery, The Second Affiliated Hospital of Xi'an Jiaotong University, Xi'an, 710004 Shaanxi, China
}

Correspondence should be addressed to Jing He; hejing198374@gmail.com and Jiwen Cheng; chengjiwen68@163.com

Received 15 March 2019; Accepted 30 April 2019; Published 25 June 2019

Academic Editor: Kum Kum Khanna

Copyright ( 2019 Jiabin Liu et al. This is an open access article distributed under the Creative Commons Attribution License, which permits unrestricted use, distribution, and reproduction in any medium, provided the original work is properly cited.

\begin{abstract}
Neuroblastoma is a life-threatening extracranial solid tumor, preferentially occurring in children. However, its etiology remains unclear. APEX1 is a critical gene in the base excision repair (BER) system responsible for maintaining genome stability. Given the potential effects of APEX1 polymorphisms on the ability of the DNA damage repair, many studies have investigated the association between these variants and susceptibility to several types of cancer but not neuroblastoma. Here, we conducted a three-center case-control study to evaluate the association between APEX1 polymorphisms ( $r s 1130409 \mathrm{~T}>\mathrm{G}, \mathrm{rs} 1760944 \mathrm{~T}>\mathrm{G}$, and rs3136817 T>C) and neuroblastoma risk in Chinese children, consisting of 469 cases and 998 controls. Odds ratio (OR) and 95\% confidence intervals (CIs) were calculated to evaluate the associations. No significant association with neuroblastoma risk was found for the studied APEX1 polymorphisms in the single locus or combination analysis. Interestingly, stratified analysis showed that rs 1130409 GG genotype significantly reduced the risk of tumor in males. Furthermore, we found that carriers with 1-3 protective genotypes had a lower neuroblastoma risk in the children older than 18 months and male, when compared to those without protective genotypes. In summary, our data indicate that APEX1 gene polymorphisms may have a weak effect on neuroblastoma susceptibility. These findings should be further validated by well-designed studies with larger sample size.
\end{abstract}

\section{Introduction}

Neuroblastoma is the most frequently diagnosed extracranial pediatric malignancy in children younger than 12 months, which accounts for almost $10 \%$ of all pediatric malignancies and $15 \%$ of pediatric cancer-related deaths [1]. Neuroblastoma is a highly heterogeneous tumor with a wide range of clinical symptoms. For instance, some patients with innocent tumors have spontaneous regression, while others have poor prognosis even after receiving intensive treatment because of the distant metastasis [2]. Overall, neuroblastoma can be classified into low-, intermediate-, and high-risk groups depending on the clinical feature, pathological phenotypes, and prognostic factors [3]. Despite the great progresses made in multimode treatments for neuroblastoma, the survival rate remains unsatisfying. The overall 5-year survival rate is around 70\%; however, the survival rate for high-risk patients is lower than $40 \%$ [4]. This poor prognosis may be partially attributed to widespread metastasis at the time of diagnosis [5].

Familial neuroblastoma with driver germline mutation is rare, accounting for approximately $1-2 \%$ of all cases [6]. The genetic alterations in the $P H O X 2 B$ and $A L K$ genes play an important role in familial neuroblastoma [7, 8]. However, the genetic mechanism of the sporadic neuroblastoma is poorly understood. Some environmental factors have been 
proposed as potential risk factors, such as maternal medication use, dwelling condition, childhood infections, conception, and pregnancy exposures $[9,10]$. To date, a direct link between neuroblastoma and environmental factors is still missing. Excitingly, the progresses in the understanding of human genome and genotyping technologies have made the genome-wide association study (GWAS) a powerful tool to study inherited genetic variations, which are associated with complex human diseases (e.g., cancer) [11]. The first neuroblastoma GWAS was performed by Maris et al. in 2008. They found that CASC15 gene polymorphisms were significantly related to neuroblastoma susceptibility [12]. Later on, the same group reported that common variants in BARD1 gene were associated with high-risk neuroblastoma [13], and polymorphisms within DUSP12, DDX4, IL31RA, and HSD17B12 were associated with low-risk neuroblastoma [14]. In 2011, Wang et al. demonstrated that LMO1 gene polymorphisms could alter the neuroblastoma susceptibility [15]. Diskin et al. proved that polymorphisms in LIN28B and HACE1 genes were also able to alter susceptibility to neuroblastoma [16]. Furthermore, the association of the polymorphisms in TP53 [17] and MMP20 [18] genes with the neuroblastoma risk was also discovered by recent GWASs successively. In addition, candidate gene approaches have been useful in identifying potential variants associated with neuroblastoma. For instance, Capasso et al. found that common variants in NEFL were associated with neuroblastoma susceptibility with 2101 neuroblastoma cases and 4202 controls of Caucasian ancestry as well as 459 neuroblastoma patients and 809 cancer-free controls of Italian descent [19]. They also discover a functional variant in the $C D K N 1 B$ gene modifying neuroblastoma susceptibility [20]. Moreover, several other predisposing genes including BARD1 [21], FAS and FASL [22], XPG [23], HOTAIR [24], and ERCC1/XPF [25] have been discovered through candidate gene approaches.

The human genome continuously suffers from DNA damages caused by exogenous (e.g., ultraviolet light, ionizing radiation chemicals) and endogenous (intracellular hydrolysis and metabolic by-products) factors. For example, the reactive oxygen species can give rise to oxidant-induced base lesions [26], which may eventually lead to genomic instability and increase tumor susceptibility if not repaired accurately. Hence, the DNA repair systems play important roles in maintaining the genomic integrity and cellular normal physiological function [27].

Base excision repair (BER) is one of the most important repair mechanisms for DNA lesions. It is widely accepted that BER is the primary pathway for repairing numerous oxidized and alkylated bases [28]. BER maintains genome integrity through recognition and excision of damaged bases. The protein product of the apurinic/apyrimidinic endonuclease 1 (APEX1) gene is one of critical enzymes in this DNA repair pathway [29]. It is reasonable to speculate that functional single nucleotide polymorphisms (SNPs) in APEX1 may affect DNA repair ability by affecting expression or function of the gene, which may further cause cell dysfunction, mutagenesis, and eventually tumorigenesis [30]. APEX1 gene polymorphisms have been shown to associate with colorectal cancer [31], cervical cancer [32], and ovarian cancer [33]. However, no publications have reported the associations of APEX1 gene polymorphisms with neuroblastoma risk. Therefore, we performed this three-center casecontrol study to assess such association in Chinese children.

\section{Materials and Methods}

2.1. Study Population. In the current three-center casecontrol research, a total of 469 histopathologically diagnosed neuroblastoma cases and 998 cancer-free controls of Chinese origin were recruited (Supplemental Table 1). Among them, 275 cases and 531 controls were recruited from Guangzhou [34-37], 118 cases and 281 controls from Zhengzhou [38-40], and the remaining 76 cases and 281 controls from Xi'an [41]. Informed consent was signed by the parents or guardians of all participants. The research protocol was authorized by the institutional review boards of corresponding institutions.

2.2. Polymorphism Selection and Genotyping. Potential functional polymorphisms in the APEX1 gene were identified through the dbSNP database (http://www.ncbi.nlm.nih.gov/) and SNPinfo (http://snpinfo.niehs.nih.gov/) [42]. Briefly, we searched the SNPs located in the untranslated regions, two terminals, and exon of the APEX1 gene. Additional selection criteria were referred from our previous publication [43]. Three SNPs (rs1130409 T>G, rs1760944 T>G, and rs3136817 T>C) in the APEX1 gene were eventually selected. As shown in Supplemental Figure 1, there is no significant linkage disequilibrium $\left(R^{2}<0.8\right)$ among these three included SNPs for Chinese Han subjects $\left(R^{2}=0.130\right.$ between rs1760944 and rs3136817, $R^{2}=0.076$ between rs1760944 and rs1130409, and $R^{2}=0.131$ between rs3136817 and rs1130409). Genomic DNA was extracted from venous blood of participants applying the TIANamp Blood DNA Kit (TIANGEN Biotech Co. Ltd., Beijing, China). Then, the purified DNA samples were genotyped for the selected polymorphisms by the standard TaqMan real-time PCR [44-47]. To ensure the reliability of the results, $10 \%$ of the DNA samples were chosen randomly for a second-time genotyping. All duplicate samples receive a concordance rate of $100 \%$.

2.3. Statistical Analysis. Deviation from the Hardy-Weinberg equilibrium (HWE) for the selected SNPs in all controls was checked by a goodness-of-fit $\chi^{2}$ test. A two-sided chi-square test was used to compare distributions of demographics and allele frequencies between all cases and controls. By unconditional logistic regression analysis, odds ratios (ORs) and 95\% confidence intervals (CIs) were calculated for assessing the association between APEX1 polymorphisms and neuroblastoma risk. Furthermore, we conducted stratified analysis according to age, gender, tumor origin site, and clinical stage. All statistical analyses were performed by applying version 9.4 SAS software (SAS Institute, NC, USA). The $P$ values $<0.05$ were thought to be statistically significant. 
TABLE 1: Association between APEX1 gene polymorphisms and neuroblastoma risk.

\begin{tabular}{|c|c|c|c|c|c|c|c|}
\hline Genotype & $\begin{array}{c}\text { Cases } \\
(N=469)\end{array}$ & $\begin{array}{c}\text { Controls } \\
(N=997)^{\mathrm{a}}\end{array}$ & $P^{\mathrm{b}}$ & $\begin{array}{c}\text { Crude OR } \\
(95 \% \text { CI })\end{array}$ & $P$ & $\begin{array}{l}\text { Adjusted OR } \\
(95 \% \mathrm{CI})^{\mathrm{c}}\end{array}$ & $P^{\mathrm{c}}$ \\
\hline \multicolumn{8}{|c|}{ rs1130409 $(\mathrm{HWE}=0.190)$} \\
\hline $\mathrm{TT}$ & $175(37.31)$ & $340(34.10)$ & & 1.00 & & 1.00 & \\
\hline TG & $216(46.06)$ & $467(46.84)$ & & $0.90(0.70-1.15)$ & 0.389 & $0.90(0.70-1.15)$ & 0.390 \\
\hline GG & $78(16.63)$ & $190(19.06)$ & & $0.80(0.58-1.10)$ & 0.167 & $0.79(0.58-1.09)$ & 0.159 \\
\hline Additive & & & 0.367 & $0.89(0.77-1.04)$ & 0.157 & $0.89(0.76-1.04)$ & 0.151 \\
\hline Dominant & $294(62.69)$ & $657(65.90)$ & 0.230 & $0.87(0.69-1.09)$ & 0.230 & $0.87(0.69-1.09)$ & 0.226 \\
\hline Recessive & $391(83.37)$ & $807(80.94)$ & 0.262 & $0.85(0.63-1.13)$ & 0.263 & $0.84(0.63-1.13)$ & 0.250 \\
\hline \multicolumn{8}{|c|}{$\mathrm{rs} 1760944(\mathrm{HWE}=0.231)$} \\
\hline $\mathrm{TT}$ & $155(33.05)$ & $334(33.50)$ & & 1.00 & & 1.00 & \\
\hline TG & $230(49.04)$ & $470(47.14)$ & & $1.05(0.82-1.35)$ & 0.674 & $1.06(0.83-1.35)$ & 0.665 \\
\hline GG & $84(17.91)$ & $193(19.36)$ & & $0.94(0.68-1.29)$ & 0.694 & $0.94(0.68-1.29)$ & 0.700 \\
\hline Additive & & & 0.736 & $0.98(0.84-1.15)$ & 0.802 & $0.98(0.84-1.15)$ & 0.810 \\
\hline Dominant & $314(66.95)$ & $663(66.50)$ & 0.864 & $1.02(0.81-1.29)$ & 0.864 & $1.02(0.81-1.29)$ & 0.854 \\
\hline Recessive & $385(82.09)$ & $804(80.64)$ & 0.509 & $0.91(0.69-1.21)$ & 0.509 & $0.91(0.69-1.21)$ & 0.511 \\
\hline \multicolumn{8}{|c|}{ rs3136817 $(\mathrm{HWE}=0.783)$} \\
\hline $\mathrm{TT}$ & $396(84.43)$ & $815(81.75)$ & & 1.00 & & 1.00 & \\
\hline $\mathrm{TC}$ & $67(14.29)$ & $172(17.25)$ & & $0.80(0.59-1.09)$ & 0.158 & $0.80(0.59-1.09)$ & 0.163 \\
\hline $\mathrm{CC}$ & $6(1.28)$ & $10(1.00)$ & & $1.24(0.45-3.42)$ & 0.685 & $1.23(0.44-3.41)$ & 0.692 \\
\hline Additive & & & 0.329 & $0.87(0.66-1.14)$ & 0.300 & $0.87(0.66-1.14)$ & 0.306 \\
\hline Dominant & $73(15.57)$ & $182(18.25)$ & 0.205 & $0.83(0.61-1.11)$ & 0.206 & $0.83(0.62-1.11)$ & 0.211 \\
\hline Recessive & $463(98.72)$ & $987(99.00)$ & 0.635 & $1.28(0.46-3.54)$ & 0.636 & $1.27(0.46-3.52)$ & 0.644 \\
\hline \multicolumn{8}{|c|}{ Combined effect of protective genotypes ${ }^{\mathrm{d}}$} \\
\hline 0 & $305(65.03)$ & $598(59.98)$ & & 1.00 & & 1.00 & \\
\hline $1-3$ & $164(34.97)$ & $399(40.02)$ & 0.064 & $0.81(0.64-1.01)$ & 0.064 & $0.81(0.64-1.01)$ & 0.064 \\
\hline
\end{tabular}

OR: odds ratio; CI: confidence interval; HWE: Hardy-Weinberg equilibrium. ${ }^{\mathrm{a} O n e}$ was failed in genotyping. ${ }^{\mathrm{b}}$ The $\chi^{2}$ test for genotype distributions between neuroblastoma patients and cancer-free controls. 'Adjusted for age and gender. ${ }^{\mathrm{d}}$ Protective genotypes were rs1130409 GG, rs1760944 GG, and $\mathrm{rs} 3136817 \mathrm{TC} / \mathrm{TT}$.

\section{Results}

3.1. Associations between APEX1 Polymorphisms and Neuroblastoma Risk. In general, this three-center casecontrol study contains 469 cases and 998 controls, of which genotyping was successfully performed in 469 cases and 997 controls. As shown in Table 1, the observed genotype frequencies of the three polymorphisms are consistent with HWE in control subjects (rs1130409: $\mathrm{HWE}=0.190$, rs1760944: $\mathrm{HWE}=0.231$, and rs3136817: $\mathrm{HWE}=0.783$ ). Unfortunately, after adjusting for age and gender, we failed to find significant association between the selected APEX1 polymorphisms and neuroblastoma risk in the single locus analysis among combined subjects (Table 1) and among subjects from each center (Supplemental Table 2). We further assessed the combined effect of protective polymorphisms of APEX1 gene on neuroblastoma risk. Subjects carrying 1-3 combined protective genotypes of APEX1 have a lower risk of neuroblastoma when compared with those without a protective genotype, though not statistically significant (adjusted OR $=0.81,95 \% \mathrm{CI}=0.64$ 1.01, $P=0.064$ ) (Table 1).
3.2. Stratification Analysis. To evaluate the effects of selected APEX1 polymorphisms on neuroblastoma risk among different subgroups, stratified analysis was conducted based on the age, gender, site of tumor origin, and clinical stage (Table 2). Null correlations were found between two polymorphisms (rs1760944 $\mathrm{T}>\mathrm{G}$ and $\mathrm{rs3136817} \mathrm{T}>\mathrm{C}$ ) and neuroblastoma risk. Interestingly, we detected that subjects with rs1130409 GG genotype have significantly decreased neuroblastoma risk in the male subgroup (adjusted $\mathrm{OR}=0.67,95 \% \mathrm{CI}=$ 0.44-0.995, $P=0.047$ ) compared with the reference group. To assess the cumulative effects of protective genotypes among subgroups, we further performed a combined analysis. Results show that subjects harboring 1-3 protective genotypes had a significantly reduced neuroblastoma risk in the following subgroups: age $>18$ months (adjusted $\mathrm{OR}=0.70$, $95 \%=0.52-0.94, P=0.016$ ) and males (adjusted $\mathrm{OR}=0.64$, $95 \% \mathrm{CI}=0.47-0.87, P=0.005)$.

\section{Discussion}

With an aim to investigate the effect of $A P E X 1$ gene polymorphisms on neuroblastoma risk, we performed this current 


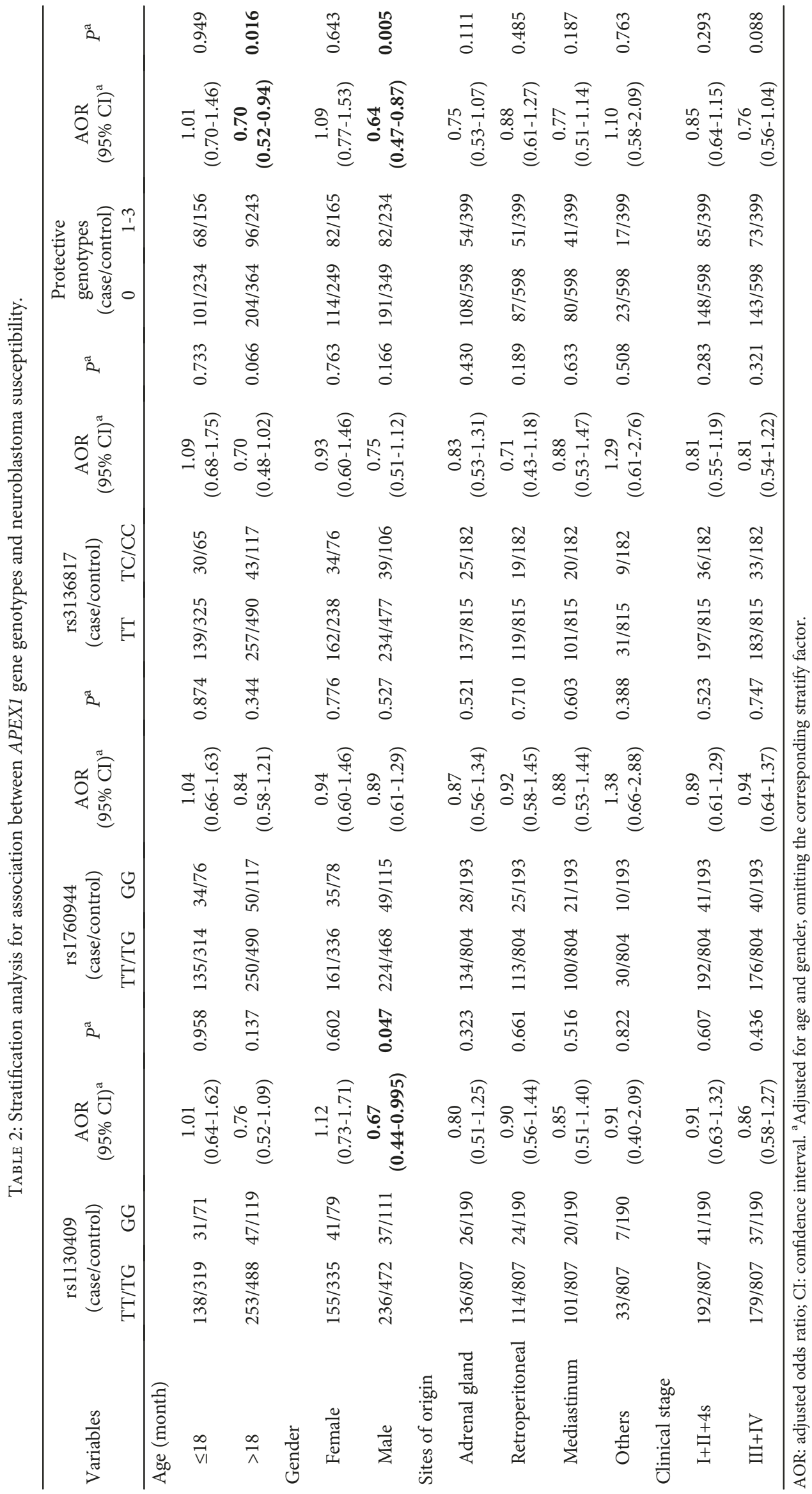


three-center case-control study in Chinese population. Neither $\mathrm{rs} 1760944 \mathrm{~T}>\mathrm{G}$ nor $\mathrm{rs} 3136817 \mathrm{~T}>\mathrm{C}$ significantly modified neuroblastoma susceptibility. However, it is noteworthy that rs1130409 GG genotype was significantly associated with reduced neuroblastoma risk in the male subgroup.

The APEX1 gene is mapped to chromosome 14q11.2-q12 and comprises five exons and four introns with $2.21 \mathrm{~kb}$ length. It encodes a nuclease acting as a rate-limiting enzyme in the BER pathway [48]. When exposed to endogenous and exogenous mutagens, apurinic/apyrimidinic (AP) sites are produced in DNA. APEX1 can hydrolyze a $5^{\prime}$-phosphodiester bond at the AP site, thereby protecting cells against the accumulation of AP sites in DNA [49]. In addition, APEX1 can act as $3^{\prime}$-phosphodiesterase which could hydrolyze $3^{\prime}$ blocking fragments of oxidized DNA, thus producing normal nucleotide $3^{\prime}$-droxyl terminals. These ends are requisite for DNA repair synthesis and ligation of gaps generated from single- or double-strand breaks [50, 51]. APEX1 is endonuclease and phosphodiesterase in the BER pathway. Polymorphisms in the APEX1 gene may alter gene expression so as to affect the activity of DNA repair and the accumulation of damaged DNA, eventually leading to carcinogenesis.

Variants in the APEX1 gene are found to associate with susceptibility to several cancers, including cervical cancer [32], glioblastoma [52], bladder cancer [53], and lung cancer $[49,54]$. To date, many SNPs in the APEX1 gene have been identified [55]. Among them, polymorphisms rs1130409 $\mathrm{T}>\mathrm{G}$ and rs1760944 $\mathrm{T}>\mathrm{G}$ were extensively studied. Polymorphism rs1130409 $\mathrm{T}>\mathrm{G}$ in the fifth exon leads to Asp148Glu residue transition in the carboxy-terminus but has no direct effect on DNA-binding and endonuclease activities of APEX1. However, polymorphism rs1760944 $\mathrm{T}>\mathrm{G}$ in the promoter region may be a pathogenic SNP associated with cancer risk [54]. The rs1130409 has been widely explored in a wide spectrum of cancers, such as bladder cancer [53]. De Ruyck et al. suggested that rs1130409 $\mathrm{T}>\mathrm{G}$ increased lung cancer risk in Caucasians [56], but Deng et al. found that this polymorphism reduced the susceptibility to lung cancer in Asians [57]. The rs1760944 T>G, one of the most frequently investigated SNPs in the APEX1 gene, is also related to cancer susceptibility, such as nasopharyngeal carcinoma (NPC) [55] and glioblastoma [52]. Lo et al. suggested that patients with genotype rs1760944 GT or GG had lower lung cancer risk compared with those with rs1760944 TT [49]. One study performed by Kang et al. showed that the genotype of rs 1760944 GG or GT may act as a protective genotype for breast cancer [58]. Li et al. found that genotype rs1760944 GG was associated with reduced risk of NPC compared with other genotypes, but the results did not reach statistical significance. More recently, one study conducted by $\mathrm{Lu}$ et al. confirmed that individuals with genotype rs1760944 GT or GG had significantly decreased NPC susceptibility than those with genotype rs1760944 TT [59]. These findings indicated that rs1760944 $\mathrm{G}$ allele may exert protective effects against several cancers. In addition, rs3136817 $\mathrm{T}>\mathrm{C}$ is one of the polymorphisms in the APEX1 gene which is investigated relatively less frequently. However, there are still some reports about association between polymorphism rs3136817 $\mathrm{T}>\mathrm{C}$ and cancer risk. For instance, Zhu et al. showed that rs3136817
TC genotype of the APEX1 gene was associated with lower risk of bladder cancer [60]. However, a research performed by $\mathrm{Li}$ et al. revealed null correlation between APEX1 rs3136817 T>C and risk of severe radiation-induced pneumonitis after radiotherapy among patients with lung cancer [61]. A recent study conducted by Wang et al. indicated that the rs3136817 TC heterozygous genotype of APEX1 has a potential protective effect for breast cancer, which may be owing to positive effect of rs3136817 TC genotype on DNA repair activity [62]. The conflicting association between APEX1 gene polymorphisms and cancer risk may be explained by the differences in the sample sizes, race, cancer types, selection criteria for subjects, and environmental exposures. Therefore, the conclusion associated between APEX1 polymorphisms and cancer risk should be specified in a particular cancer type and population.

Herein, we for the first time investigated the association between the APEX1 gene SNPs and neuroblastoma risk. No significant association was found in the single locus analysis or combined analysis. Several reasons may help to explain the unexpected negative results: (1) the role of a polymorphism in cancer susceptibility might be tissueand ethnicity-dependent and (2) the sample size of this study was too small to detect a real association. Although no significant effect was found in the overall analysis, age and gender may modify the results. In the stratified analysis, we found that rs1130409 GG genotype significantly decreased the risk of neuroblastoma in the male subgroup. To date, the rs1130409 $\mathrm{T}>\mathrm{G}$ of the APEX1 gene is the most extensively studied SNP. Moreover, we also found that the presence of 1-3 protective genotypes provided a protective effect for children $>18$ months of age and boys. It should be noted that these significant results may be just chance findings in the stratification analysis because of relatively small sample size.

There are several accompanied limitations. First, although subjects in this current study were recruited from three independent hospitals, the sample size remains moderate, especially for the stratified analysis. As a result, the statistical powers were ineluctably whittled. Second, only three polymorphisms in the APEX1 gene were investigated. Other potentially functional APEX1 polymorphisms should be assessed. Third, the subjects involved in this study are Chinese; therefore, the conclusions obtained from this study may not apply to other ethnicities. Fourth, only genetic analysis was performed in neuroblastoma risk. Other environmental factors such as living environment, dietary habits, and childhood or parental exposure should be considered, as neuroblastoma is a heterogeneous disease with a complicated etiology.

\section{Conclusions}

In conclusion, our present data suggest that APEX1 polymorphisms affect neuroblastoma susceptibility in a lowpenetrance manner. Well-designed multicenter studies with larger sample size are needed to confirm our findings. Furthermore, functional validation should be performed to clarify the underlying mechanisms by which APEX1 gene polymorphisms affect neuroblastoma susceptibility. 

Abbreviations
GWAS: Genome-wide association study
BER: $\quad$ Base excision repair
APEX1: Apuriniclapyrimidinic endonuclease 1
SNP: $\quad$ Single nucleotide polymorphism
HWE: Hardy-Weinberg equilibrium
OR: Odds ratio
CI: Confidence interval.

\section{Data Availability}

The data used to support the findings of this study are available from the corresponding author upon request.

\section{Conflicts of Interest}

The authors declare that there is no conflict of interests regarding the publication of this paper.

\section{Authors' Contributions}

HX, JC, and JH conceived and designed the study. JC, J Zhang, TY, PL, and JH collected samples. JH provided technical support. JL, WJ, and RXH collected and processed data and conducted the experiment. $\mathrm{JH}$ analyzed data and prepared tables. JL, J Zhu, and JH wrote the manuscript. All authors approved the final manuscript. Jiabin Liu, Wei Jia, and Rui-Xi Hua contributed equally to this work.

\section{Acknowledgments}

This work was supported by grants from the Pearl River S and T Nova Program of Guangzhou (No. 201710010086), the National Natural Science Foundation of China (No. 81602199), the Guangzhou Science, Technology and Innovation Commission (No. 201607010395), and the Natural Science Foundation of Guangdong Province, China (No. 2016A030313496).

\section{Supplementary Materials}

Supplemental Table 1: frequency distribution of selected characteristics in cases and controls. Supplemental Table 2: association between APEX1 gene polymorphisms and neuroblastoma risk (divided subjects). Supplemental Figure 1: linkage disequilibrium analysis for the three selected polymorphisms in the APEX1 gene in the Han Chinese population consisting of CHS (Southern Han Chinese) and CHB (Han Chinese in Beijing, China) subjects using data from LDlink online software (https://dlink.nci.nih.gov/ ?tab=ldmatrix). (Supplementary Materials)

\section{References}

[1] K. K. Matthay, J. M. Maris, G. Schleiermacher et al., "Neuroblastoma," Nature Reviews Disease Primers, vol. 2, no. 1, article 16078, 2016.
[2] N.-K. V. Cheung and M. A. Dyer, "Neuroblastoma: developmental biology, cancer genomics and immunotherapy," Nature Reviews. Cancer, vol. 13, no. 6, pp. 397-411, 2013.

[3] H. Shimada, I. M. Ambros, L. P. Dehner et al., "The International Neuroblastoma Pathology Classification (the Shimada system)," Cancer, vol. 86, no. 2, pp. 364-372, 1999.

[4] F. Berthold, J. Boos, S. Burdach et al., "Myeloablative megatherapy with autologous stem-cell rescue versus oral maintenance chemotherapy as consolidation treatment in patients with high-risk neuroblastoma: a randomised controlled trial," The Lancet Oncology, vol. 6, no. 9, pp. 649-658, 2005.

[5] M. R. Esposito, S. Aveic, A. Seydel, and G. P. Tonini, "Neuroblastoma treatment in the post-genomic era," Journal of Biomedical Science, vol. 24, no. 1, p. 14, 2017.

[6] M. Capasso and S. J. Diskin, "Genetics and genomics of neuroblastoma," Cancer Treatment and Research, vol. 155, pp. 65-84, 2010.

[7] D. Trochet, F. Bourdeaut, I. Janoueix-Lerosey et al., "Germline mutations of the paired-like homeobox 2B (PHOX2B) gene in neuroblastoma," American Journal of Human Genetics, vol. 74, no. 4, pp. 761-764, 2004.

[8] Y. P. Mossé, M. Laudenslager, L. Longo et al., "Identification of ALK as a major familial neuroblastoma predisposition gene," Nature, vol. 455, no. 7215, pp. 930-935, 2008.

[9] M. N. Cook, A. F. Olshan, H. A. Guess et al., "Maternal medication use and neuroblastoma in offspring," American Journal of Epidemiology, vol. 159, no. 8, pp. 721-731, 2004.

[10] F. Menegaux, A. F. Olshan, J. P. Neglia, B. H. Pollock, and M. L. Bondy, "Day care, childhood infections, and risk of neuroblastoma," American Journal of Epidemiology, vol. 159, no. 9, pp. 843-851, 2004.

[11] K. A. Frazer, S. S. Murray, N. J. Schork, and E. J. Topol, "Human genetic variation and its contribution to complex traits," Nature Reviews. Genetics, vol. 10, no. 4, pp. 241-251, 2009.

[12] J. M. Maris, Y. P. Mosse, J. P. Bradfield et al., "Chromosome 6 p22 locus associated with clinically aggressive neuroblastoma," The New England Journal of Medicine, vol. 358, no. 24, pp. 2585-2593, 2008.

[13] M. Capasso, M. Devoto, C. Hou et al., "Common variations in BARD1 influence susceptibility to high-risk neuroblastoma," Nature Genetics, vol. 41, no. 6, pp. 718-723, 2009.

[14] L. B. Nguyễn, S. J. Diskin, M. Capasso et al., "Phenotype restricted genome-wide association study using a genecentric approach identifies three low-risk neuroblastoma susceptibility loci," PLoS Genetics, vol. 7, no. 3, article e1002026, 2011.

[15] K. Wang, S. J. Diskin, H. Zhang et al., "Integrative genomics identifies LMO1 as a neuroblastoma oncogene," Nature, vol. 469, no. 7329, pp. 216-220, 2011.

[16] S. J. Diskin, M. Capasso, R. W. Schnepp et al., "Common variation at $6 \mathrm{q} 16$ within HACE1 and LIN28B influences susceptibility to neuroblastoma," Nature Genetics, vol. 44, no. 10, pp. 1126-1130, 2012.

[17] S. J. Diskin, M. Capasso, M. Diamond et al., "Rare variants in TP53 and susceptibility to neuroblastoma," Journal of the National Cancer Institute, vol. 106, no. 4, article dju047, 2014.

[18] X. Chang, Y. Zhao, C. Hou et al., "Common variants in MMP20 at 11q22.2 predispose to $11 \mathrm{q}$ deletion and neuroblastoma risk," Nature Communications, vol. 8, no. 1, p. 569, 2017. 
[19] M. Capasso, S. Diskin, F. Cimmino et al., "Common genetic variants in NEFL influence gene expression and neuroblastoma risk," Cancer Research, vol. 74, no. 23, pp. 6913-6924, 2014.

[20] M. Capasso, L. D. McDaniel, F. Cimmino et al., "The functional variant rs34330 of CDKN1B is associated with risk of neuroblastoma," Journal of Cellular and Molecular Medicine, vol. 21, no. 12, pp. 3224-3230, 2017.

[21] F. Cimmino, M. Avitabile, S. J. Diskin et al., "Fine mapping of 2q35 high-risk neuroblastoma locus reveals independent functional risk variants and suggests full-length BARD1 as tumor-suppressor," International Journal of Cancer, vol. 143, no. 11, pp. 2828-2837, 2018.

[22] W. Han, Y. Zhou, R. Zhong et al., "Functional polymorphisms in FAS/FASL system increase the risk of neuroblastoma in Chinese population," PLoS One, vol. 8, no. 8, article e71656, 2013.

[23] J. He, F. Wang, J. Zhu et al., "Association of potentially functional variants in the XPG gene with neuroblastoma risk in a Chinese population," Journal of Cellular and Molecular Medicine, vol. 20, no. 8, pp. 1481-1490, 2016.

[24] X. Yang, J. He, Y. Chang et al., "HOTAIR gene polymorphisms contribute to increased neuroblastoma susceptibility in Chinese children," Cancer, vol. 124, no. 12, pp. 2599-2606, 2018.

[25] Z. J. Zhuo, W. Liu, J. Zhang et al., "Functional polymorphisms at ERCC1/XPF genes confer neuroblastoma risk in Chinese children," eBioMedicine, vol. 30, pp. 113-119, 2018.

[26] A. M. Whitaker, M. A. Schaich, M. R. Smith, T. S. Flynn, and B. D. Freudenthal, "Base excision repair of oxidative DNA damage: from mechanism to disease," Frontiers in Bioscience, vol. 22, pp. 1493-1522, 2017.

[27] R. D. Wood, M. Mitchell, J. Sgouros, and T. Lindahl, "Human DNA repair genes," Science, vol. 291, no. 5507, pp. 1284-1289, 2001.

[28] A. Barzilai and K. I. Yamamoto, "DNA damage responses to oxidative stress," DNA Repair, vol. 3, no. 8-9, pp. 1109-1115, 2004.

[29] R. J. Hung, J. Hall, P. Brennan, and P. Boffetta, "Genetic polymorphisms in the base excision repair pathway and cancer risk: a HuGE review," American Journal of Epidemiology, vol. 162, no. 10, pp. 925-942, 2005.

[30] H. Tan, "The association between gene SNPs and cancer predisposition: correlation or causality?," eBioMedicine, vol. 16, pp. 8-9, 2017.

[31] Y. Li, S. Li, Z. Wu et al., "Polymorphisms in genes of APE1, PARP1, and XRCC1: risk and prognosis of colorectal cancer in a northeast Chinese population," Medical Oncology, vol. 30, no. 2, p. 505, 2013.

[32] X. F. Wang, M. Z. Huang, X. W. Zhang, R. X. Hua, and W. J. Guo, "COX-2-765G $>$ C polymorphism increases the risk of cancer: a meta-analysis," PLoS One, vol. 8, no. 9, article e73213, 2013

[33] X. Zhang, X. Xin, J. Zhang, J. Li, B. Chen, and W. Zou, "Apurinic/apyrimidinic endonuclease 1 polymorphisms are associated with ovarian cancer susceptibility in a Chinese population," International Journal of Gynecological Cancer, vol. 23 , no. 8 , pp. 1393-1399, 2013.

[34] J. He, Y. Zou, X. Liu et al., "Association of common genetic variants in pre-microRNAs and neuroblastoma susceptibility: a two-center study in Chinese children," Molecular Therapy Nucleic Acids, vol. 11, pp. 1-8, 2018.
[35] J. Tang, W. Liu, J. Zhu et al., "RSRC1 and CPZ gene polymorphisms with neuroblastoma susceptibility in Chinese children," Gene, vol. 662, pp. 83-87, 2018.

[36] J. Tao, Z. J. Zhuo, M. Su, L. Yan, J. He, and J. Zhang, "XPA gene polymorphisms and risk of neuroblastoma in Chinese children: a two-center case-control study," Journal of Cancer, vol. 9, no. 15, pp. 2751-2756, 2018.

[37] Q. Wu, Z. J. Zhuo, J. Zeng et al., “Association between NEFL gene polymorphisms and neuroblastoma risk in Chinese children: a two-center case-control study," Journal of Cancer, vol. 9, no. 3, pp. 535-539, 2018.

[38] J. Zhang, H. Lin, J. Wang et al., " $L M O 1$ polymorphisms reduce neuroblastoma risk in Chinese children: a two-center casecontrol study," Oncotarget, vol. 8, no. 39, pp. 65620-65626, 2017.

[39] J. Zhang, Z. J. Zhuo, J. Wang et al., "CASC15 gene polymorphisms reduce neuroblastoma risk in Chinese children," Oncotarget, vol. 8, no. 53, pp. 91343-91349, 2017.

[40] J. Zhang, Z. Zhuo, W. Li, J. Zhu, J. He, and J. Su, "XRCC1 gene polymorphisms and risk of neuroblastoma in Chinese children," Aging, vol. 10, no. 10, pp. 2944-2953, 2018.

[41] J. Cheng, Z. Zhuo, Y. Xin et al., "Relevance of XPD polymorphisms to neuroblastoma risk in Chinese children: a four-center case-control study," Aging, vol. 10, no. 8, pp. 1989-2000, 2018.

[42] J. Zhu, W. Jia, C. Wu et al., "Base excision repair gene polymorphisms and Wilms tumor susceptibility," eBioMedicine, vol. 33, pp. 88-93, 2018.

[43] J. He, L. X. Qiu, M. Y. Wang et al., "Polymorphisms in the XPG gene and risk of gastric cancer in Chinese populations," Human Genetics, vol. 131, no. 7, pp. 1235-1244, 2012.

[44] J. Gong, J. Tian, J. Lou et al., “A polymorphic MYC response element in KBTBD11 influences colorectal cancer risk, especially in interaction with an MYC-regulated SNP rs6983267," Annals of Oncology, vol. 29, no. 3, pp. 632639, 2018.

[45] J. Lou, J. Gong, J. Ke et al., “A functional polymorphism located at transcription factor binding sites, rs6695837 near LAMC1 gene, confers risk of colorectal cancer in Chinese populations," Carcinogenesis, vol. 38, no. 2, pp. 177-183, 2017.

[46] D. Zou, J. Lou, J. Ke et al., "Integrative expression quantitative trait locus-based analysis of colorectal cancer identified a functional polymorphism regulating SLC22A5 expression," European Journal of Cancer, vol. 93, pp. 1-9, 2018.

[47] J. Li, L. Zou, Y. Zhou et al., "A low-frequency variant in SMAD7 modulates TGF- $\beta$ signaling and confers risk for colorectal cancer in Chinese population," Molecular Carcinogenesis, vol. 56, no. 7, pp. 1798-1807, 2017.

[48] J. L. Parsons, I. I. Dianova, and G. L. Dianov, "APE1 is the major $3^{\prime}$-phosphoglycolate activity in human cell extracts," Nucleic Acids Research, vol. 32, no. 12, pp. 3531-3536, 2004.

[49] Y. L. Lo, Y. S. Jou, C. F. Hsiao et al., "A polymorphism in the APE1 gene promoter is associated with lung cancer risk," Cancer Epidemiology, Biomarkers \& Prevention, vol. 18, no. 1, pp. 223-229, 2009.

[50] T. Izumi, T. K. Hazra, I. Boldogh et al., "Requirement for human AP endonuclease 1 for repair of 3 '-blocking damage at DNA single-strand breaks induced by reactive oxygen species," Carcinogenesis, vol. 21, no. 7, pp. 1329-1334, 2000.

[51] M. R. Kelley, L. Cheng, R. Foster et al., "Elevated and altered expression of the multifunctional DNA base excision repair 
and redox enzyme Ape1/ref-1 in prostate cancer," Clinical Cancer Research, vol. 7, no. 4, pp. 824-830, 2001.

[52] K. Zhou, D. Hu, J. Lu et al., "A genetic variant in the APE1/Ref-1 gene promoter $-141 \mathrm{~T} / \mathrm{G}$ may modulate risk of glioblastoma in a Chinese Han population," BMC Cancer, vol. 11, no. 1, 2011.

[53] M. Wang, C. Qin, J. Zhu et al., "Genetic variants of XRCC1, $A P E 1$, and ADPRT genes and risk of bladder cancer," DNA and Cell Biology, vol. 29, no. 6, pp. 303-311, 2010.

[54] J. Lu, S. Zhang, D. Chen et al., "Functional characterization of a promoter polymorphism in APE1/Ref- 1 that contributes to reduced lung cancer susceptibility," The FASEB Journal, vol. 23, no. 10, pp. 3459-3469, 2009.

[55] Q. Li, J. M. Wang, Y. Peng et al., “Association of DNA baseexcision repair XRCC1, OGG1 and APE1 gene polymorphisms with nasopharyngeal carcinoma susceptibility in a Chinese population," Asian Pacific Journal of Cancer Prevention, vol. 14, no. 9, pp. 5145-5151, 2013.

[56] K. de Ruyck, M. Szaumkessel, I. de Rudder et al., "Polymorphisms in base-excision repair and nucleotide-excision repair genes in relation to lung cancer risk," Mutation Research, vol. 631, no. 2, pp. 101-110, 2007.

[57] Q. Deng, L. Sheng, D. Su et al., "Genetic polymorphisms in ATM, ERCC1, APE1 and iASPP genes and lung cancer risk in a population of southeast China," Medical Oncology, vol. 28, no. 3, pp. 667-672, 2011.

[58] H. Kang, Z. Dai, X. Ma et al., "A genetic variant in the promoter of APE1 gene $(-656 \mathrm{~T}>\mathrm{G})$ is associated with breast cancer risk and progression in a Chinese population," Gene, vol. 531, no. 1, pp. 97-100, 2013.

[59] Z. Lu, S. Li, S. Ning et al., "Association of the rs1760944 polymorphism in the APEX1 base excision repair gene with risk of nasopharyngeal carcinoma in a population from an endemic area in South China," Journal of Clinical Laboratory Analysis, vol. 32, no. 2, article e22238, 2018.

[60] G. Zhu, H. Su, L. Lu et al., “Association of nineteen polymorphisms from seven DNA repair genes and the risk for bladder cancer in Gansu province of China," Oncotarget, vol. 7, no. 21, pp. 31372-31383, 2016.

[61] H. Li, G. Liu, L. Xia et al., "A polymorphism in the DNA repair domain of APEX1 is associated with the radiation-induced pneumonitis risk among lung cancer patients after radiotherapy," The British Journal of Radiology, vol. 87, no. 1040, article 20140093, 2014.

[62] T. Wang, H. Wang, S. Yang et al., "Association of APEX1 and OGG1 gene polymorphisms with breast cancer risk among Han women in the Gansu Province of China," BMC Medical Genetics, vol. 19, no. 1, p. 67, 2018. 


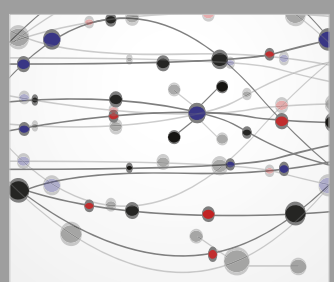

The Scientific World Journal
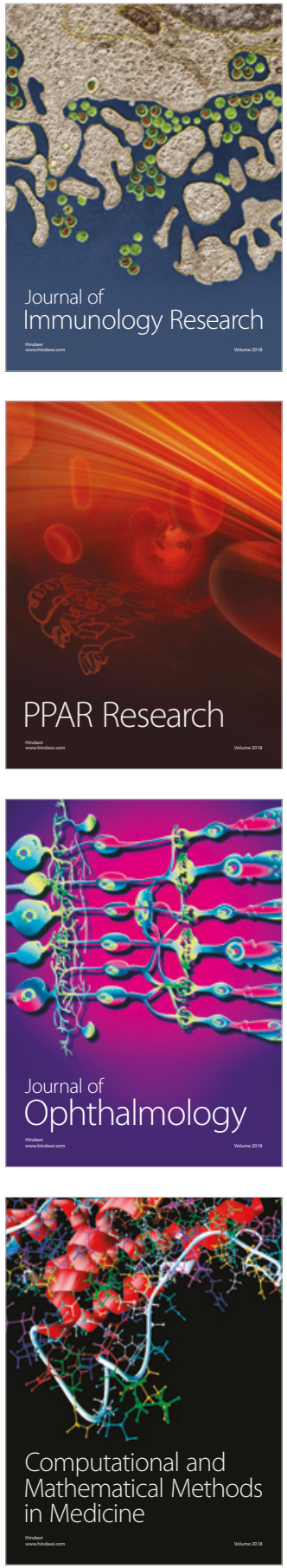

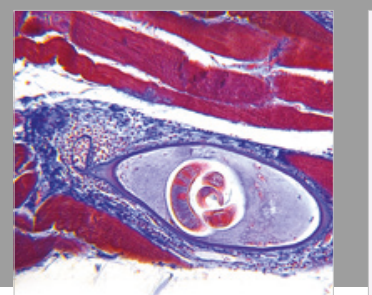

Gastroenterology Research and Practice

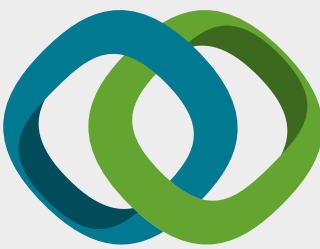

\section{Hindawi}

Submit your manuscripts at

www.hindawi.com
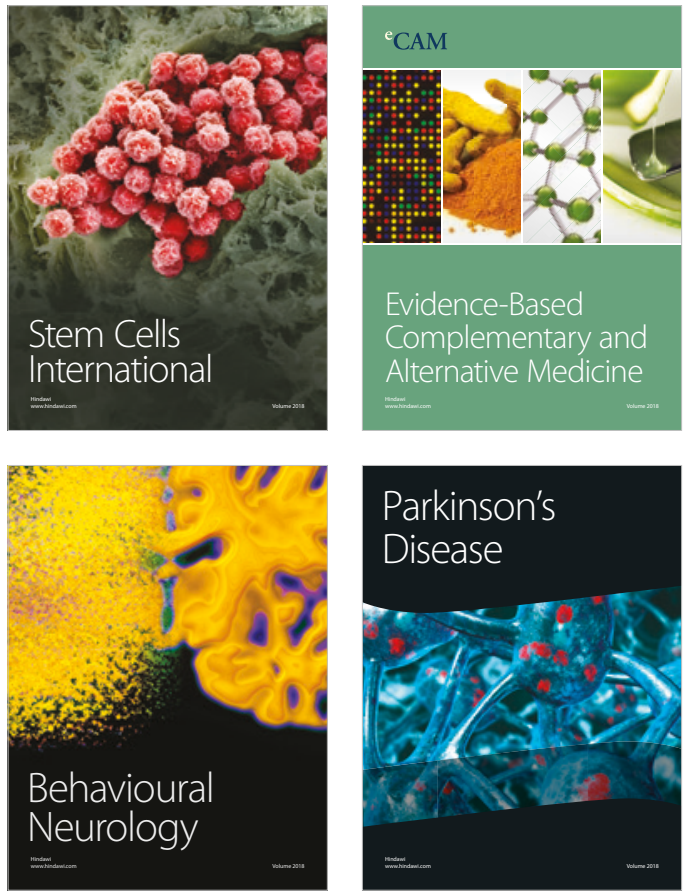

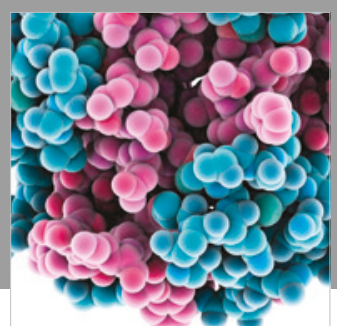

ournal of

Diabetes Research

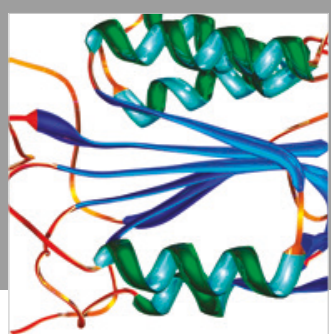

Disease Markers
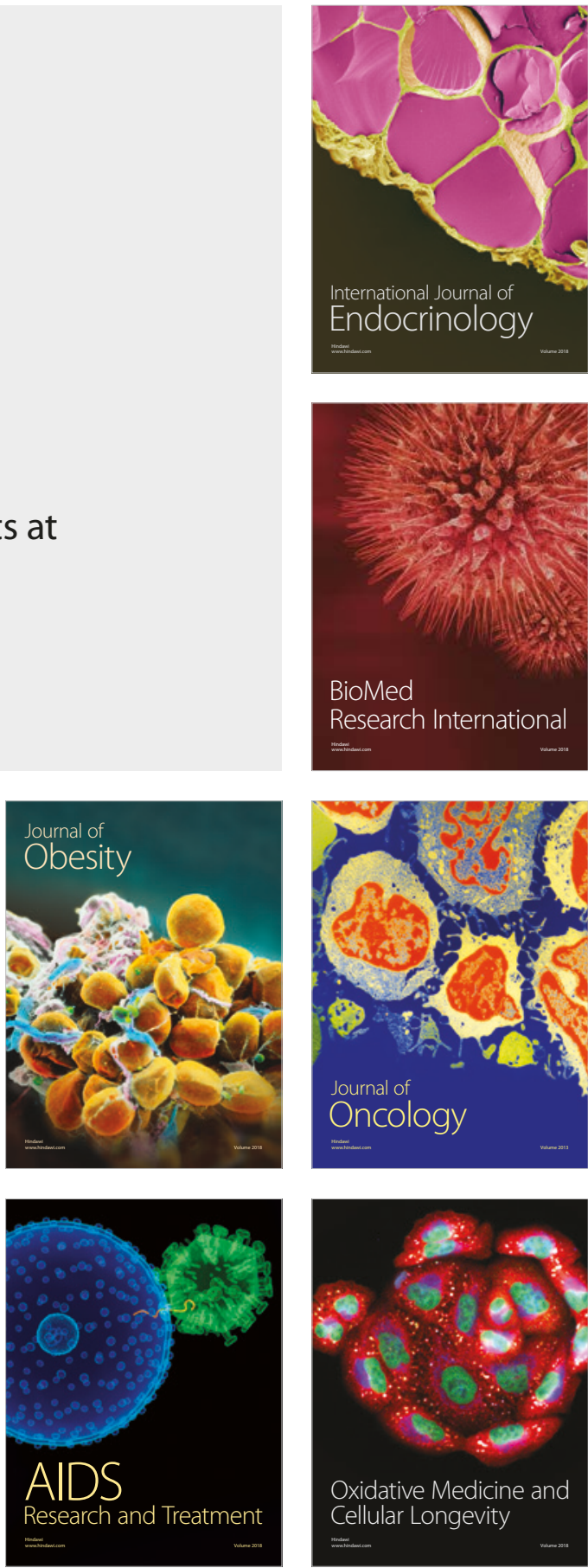